\title{
Análisis de las relaciones internacionales del Ecuador con la Comunidad Andina de Naciones (CAN) para determinar su efecto en el flujo del comercio internacional de la región
}

\author{
César E. Freire, Félix M. Carrera, Gabriela E. Hurtado* y Jessica S. Matute \\ Facultad de Ciencias Económicas y Administrativas, Univ. Católica de Santiago de Guayaquil \\ (Correo-e: cesar.freire@cu.ucsg.edu.ec; felix.carrera01@cu.ucsg.edu.ec; gabriela.hurtado@cu.ucsg.edu.ec; \\ jessica.matute@cu.uscg.edu.ec) \\ * Autor a quien debe ser enviada la correspondencia
}

Recibido May. 24, 2021; Aceptado Jul. 19, 2021; Versión final Sep. 7, 2021, Publicado Feb. 2022

\begin{abstract}
Resumen
El objetivo de esta investigación es analizar las relaciones internacionales entre países miembros de la Comunidad Andina de Naciones (CAN) y su efecto en el flujo de comercio internacional entre Ecuador y los países miembros. Se consideran dos perspectivas. La primera analiza el cambio significativo que pudieron tener las exportaciones de Ecuador hacia países miembros de la CAN. La segunda considera el cambio significativo que puedan tener las importaciones de Ecuador que provienen de países miembros de la CAN. Para modelar las series se considera un modelo ARMAX (Auto-Regressive Moving Average with exogenous inputs). Como resultado se obtiene que el flujo de importaciones ha aumentado fuertemente por parte del Ecuador. Se pronostica que la tendencia se mantenga al alta ya que los valores de la constante e intercepto de los modelos se mantienen significantes. Se concluye que el flujo de apertura comercial entre países miembros de la CAN ha tenido un dinamismo significativo, lo cual da relevancia a este tipo de acuerdos.
\end{abstract}

\section{Analysis of Ecuador's international relations with the Andean Community of Nations (CAN) to determine its effect on international trade flows in the region}

\begin{abstract}
The main objective of this research study is to examine international relations between countries of the Andean Community of Nations (CAN, in Spanish) and assess their effects on international trade flows between Ecuador and CAN members. Two perspectives are considered. The first perspective evaluates whether there are significant changes in Ecuador's exports to CAN countries. The second perspective considers significant changes, if any, in Ecuador's imports from CAN countries. To model the series, an ARMAX (Auto-Regressive Moving Average model with exogenous inputs) model is applied. The results show that import flows have increased significantly in Ecuador. It is predicted that this will remain on an upward trend since the values of the constant and the intercept of the models remain significant. It is concluded that the flow of international trade between CAN members is highly dynamic, which reinforces the importance of this type of trade agreements.
\end{abstract}




\section{INTRODUCCIÓN}

Para Hinojosa (2005) la globalización es un fenómeno que trae consigo una serie de connotaciones económicas, políticas, sociales e incluso culturales, mientras que para Molano (2017) ésta ha generado cambios y nuevas tensiones sociales en la distribución nacional e internacional de los recursos. Keohane y Nye (2000) mencionaron que la globalización genera un aumento de la interdependencia con dos características, redes de conexiones múltiples distancias multicontinentales. Se distinguen tres dimensiones de la globalización, la económica asociada al desarrollo de la revolución tecnológica e informática, conjuntamente con el accionar de multinacionales y las inversiones en el extranjero. La política, vinculada a la primacía occidental-estadounidense, así como también las organizaciones internacionales, regionales y redes transgubernamentales. Y la globalización cultural relacionada con la circulación de los productos culturales, que para algunos presentaría enormes desafíos ante la uniformidad y para otros, en cambio, potenciaría las reivindicaciones de singularidad (Hoffmann, 2002).

Held (1997) y Meyer (2012), por su parte, sostienen que con el proceso de globalización los Estados estarían perdiendo sus rasgos esenciales asociados a la capacidad de ejercer la soberanía tradicional y mantener la gobernanza. Silva (2009) mencionó que los aspectos más importantes de la dimensión económica de la globalización son: 1) comercio internacional, 2) flujos de inversión extranjera; y 3) capitales financieros. Allard (2010) señaló que la globalización, por su naturaleza, obliga a ampliar el número de actores, no solo a los Estados y organizaciones internacionales, sino también a las ONGs y las empresas multinacionales, dando a este proceso-fenómeno una amplitud y una extensión que en sus inicios habría sido difícil de imaginar.En cuanto a la negociación internacional Coronado (2001) infiere que los Estados transnacionales deben compartir las lealtades de sus súbditos con otras autoridades regionales y de la sociedad mundial, por una parte y, por la otra, con autoridades subestatales y subnacionales.

La negociación comercial internacional se desprende de la globalización económica, según Malfeito (2012) esta negociación es el resultado del deseo de los agentes económicos por ampliar actividades a lo largo del mundo mediante la libertad de movimientos de los elementos. En el ámbito de las Relaciones Internacionales, el constructivismo reconoce el poder de las instituciones multilaterales, a la vez que advierte la continuidad entre la toma de decisiones a nivel doméstico e internacional (Haas, 2006). Palomares (2006) señala que el intercambio comercial internacional se caracteriza por "un notable incremento en la capacidad de las firmas para fragmentar geográficamente los procesos productivos, lo que ha tenido como contrapartida un crecimiento sostenido del comercio y la inversión internacionales". Según Van De Mieroop (2002) el comercio y los intercambios comerciales experimentaron un auge hacia el 2600 a.C. Para Amunátegui y Carvajal (2016) el comercio requiere del desarrollo de unidades métricas que sirvan de base al intercambio y que tengan la virtualidad de ser aceptadas por todos como equivalentes a las mercancías intercambiadas.

En la complejidad de las relaciones internacionales se deben revisar varias perspectivas, tal como la perspectiva de la teoría intercultural de las relaciones internacionales basada en tres formas distintas de establecer la autoestima, honor y dignidad. La teoría explica variaciones importantes en la forma en que los estados y las naciones se relacionan con los miembros de su propia cultura; así como con los miembros de otras culturas similares (Friedrichs, 2016). Algunos teóricos preferirán pensar en la Teoría de las Relaciones Internacionales como "la explicación de las leyes que identifican rasgos permanentes o probables asociaciones entre Estados"; otros dirán que la TRI es una "tradición de especulaciones sobre las relaciones entre los Estados" (Wight); todavía las teorías empíricas preferirán remitir al "uso de la observación para la verificación de hipótesis sobre el mundo", y así podríamos seguir hasta el infinito. Dentro de la disciplina, y a sabiendas de la simplificación que supone cualquier reducción a extremos, se destacan dos énfasis en el estudio de las Relaciones Internacionales (RI). Existen autores que destacan que la tarea de las Relaciones Internacionales es explicar, analizar e interpretar eventos, estructuras e instituciones; así como también el comportamiento de los agentes que las conducen tanto de forma individual como colectiva (Lawson, 2015).

En este contexto, la presente investigación pretende conocer la manera en la cual las relaciones internacionales y la cooperación internacional ejercida por Ecuador en los últimos años ha incidido en el flujo del comercio internacional del país con sus vecinos, particularmente a partir de las exportaciones e importaciones. Una de las herramientas analíticas más útiles en RI es el "Sistema Internacional" (SI) definido como "el conjunto de interacciones entre los diferentes actores internacionales". Este SI está constituido por un conjunto de actores cuyas relaciones generan una configuración de poder (estructura), dentro de la cual se produce una red compleja de interacciones (procesos) de acuerdo a determinadas reglas (Caruncho, et al., 2003).

Debe advertirse que, tradicionalmente, en el estudio teórico de las RI han prevalecido los análisis sobre los conflictos y sus causas, relegándose los aspectos teóricos de la Cooperación Internacional a un segundo plano. Es así que posteriormente al estudio de las Relaciones Internacionalescomo disciplina científica post guerra y post conflicto, se incluyó a la Cooperación Internacional $(\mathrm{Cl})$, y más directamente en una de las 
modalidades específicas que adopta: la Cooperación Internacional para el Desarrollo.De las muchas definiciones existentes sobre Cooperación Internacional, se propone la de Calduch que considera que la $\mathrm{Cl}$ es "toda relación entre actores internacionales orientada a la mutua satisfacción de intereses o demandas, mediante la utilización complementaria de sus respectivos poderes en el desarrollo de actuaciones coordinadas y/o solidarias" (Cornago, 1998).

Aplicado al campo de las Relaciones Internacionales se define la Cl como la modalidad de cooperación que se realiza entre actores del sistema internacional. Sise aplica esta definición, ¿cuáles serían los elementos que configurarían una relación de Cooperación Internacional para que pueda ser identificada como tal? Un teórico de las RI, Holsti (1983) ofreció la respuesta. Estos elementos serían: 1) la percepción de que dos o más intereses coinciden y pueden ser alcanzados por ambas partes simultáneamente; 2) la expectativa de una de las partes de que la actuación seguida por la otra parte, o las otras partes si la cooperación fuese multilateral, en orden a lograr sus propios objetivos, le ayuda a realizar sus intereses y valores;3) la existencia de un acuerdo (expreso o tácito) sobre los aspectos esenciales de las transacciones o de las actividades a realizar; y 4)la aplicación de reglas y pautas (protocolos de actuación) que dominarán las futuras transacciones (Holsti, 1983).

Las clasificaciones sobre las categorías de las relaciones de Cooperación Internacional son muy variadas. Etimológicamente "cooperar" significa actuar conjuntamente con otros para conseguir un mismo fin. La cooperación implica coordinación de intereses a partir de la percepción de problemas comunes para los que no poseemos respuesta o capacidad individual. Idealmente supone también reciprocidad, deseos de dar y recibir, esfuerzo colectivo, creación de oportunidades para todos, distribución equitativa de costes y beneficios. La diferencia entre cooperación y ayuda es que la primera asume que se trata de un tipo de relación recíproca que conlleva formas de asociación mientras que la segunda contiene también un sentido social, pero no implica compartir pues supone la existencia previa de una desigualdad (Rodríguez \& Sotillo, 2003). No es menos cierto que no todas las relaciones de cooperación llevan aparejado el concepto de igualdad o de equilibrio. Desde luego en las relaciones internacionales resulta evidente que la igualdad en las relaciones cooperativas se da con más frecuencia cuando existen similares recursos de poder entre los participantes. A partir de este último enfoque se comprenden las situaciones de desigualdad y desequilibrio que viven algunos países miembros de algunos bloques de cooperación que poseen diferentes niveles de poder financiero, tecnológico, productivo con distintas repercusiones tanto en su crecimiento económico como en el desarrollo social de sus ciudadanos, como por ejemplo la realidad mexicana frente al Mercado del Norte, o NAFTA con sus siglas en inglés (Rogerson et al., 2004).

La cooperación se define en sentido amplio como un sistema de interacciones entre distintos actores y organizaciones cuyo objetivo es lograr unos niveles de conformidad recíproca mediante un proceso de negociación denominado coordinación de políticas (Bhansali, 1987). Se describe en la Cooperación Internacional Moderna varias etapas. Entre las principales señala como la Primera etapa a la que se desarrolla entre (1950-1965) y que se caracteriza por la visión del desarrollo a través del crecimiento por la inversión. Corresponde a la etapa de la post guerra, y fue guiada en el occidente por las teorías keynesianas. El centro de la cooperación para el desarrollo de esta etapa, fue vincularla a la inversión internacional, lo que centraba el desarrollo y la cooperación internacional a aspectos fundamentalmente económicos y financieros y como una Segunda etapa a la desarrollada entre 1965-1980, la misma que se identificaría con los conceptos de interdependencia y necesidades básicas. Surge como una respuesta a la constatación de que el esperado desarrollo de los países del sur no se había producido, entre otros motivos por su asimetría en el acceso a mercados, competitividad y dependencia. En esta evolución, la creciente evidencia sobre la importancia del lado humano del desarrollo y el creciente debate sobre las necesidades humanas, da al desarrollo y con ello a la cooperación Internacional otra perspectiva (Browne, 1997)

Algunos autores afirman que "en el origen de las relaciones cooperativas existe un cálculo individual que revela que el mejor modo de conseguir uno o más objetivos nacionales fundamentales es cooperar con otro gobierno, dando vida así a un tipo de relación particular y diferente de la que se tiene con otros gobiernos en relación con los mismos objetivos". Más allá del primer móvil que origina la cooperación entre Estados, otros se han centrado en explicitar las razones que favorecen el establecimiento de relaciones de cooperación. Según el citado (Holsti, 1983) estas son: 1) existencia de objetivos, intereses y necesidades similares o complementarias entre las partes; 2) distribución equitativa de costes, riesgos y beneficios entre las partes, confianza en que la otra parte cumplirá con sus obligaciones; y 3) las interacciones se desarrollan en términos de reciprocidad y confianza mutua (Iglesia, 2005).

La nueva teoría del comercio internacional nace a finales de 1970, y se encuentra sustentada en dos supuestos: comercio internacional y organizaciones industriales. La nueva teoría del comercio internacional demuestra que varios de los argumentos de la teoría clásica del comercio internacional han sido remplazados y mejorados por nuevos argumentos como las economías de escala; comercio intraindustrial; economías externas; competencia imperfecta; movilidad de factores, etc. 


\section{OTROS ANTECEDENTES}

En los últimos años, las teorías sobre la cooperación internacional han prestado atención a tres temas sustantivos: Ios actores no estatales (ANE), las normas e ideas transnacionales y la eficacia de la cooperación. Actores no estatales: un número un número cada vez mayor de NSA desempeña un papel importante en la cooperación internacional, incluidas las organizaciones internacionales, los movimientos sociales transnacionales, la industria privada y las comunidades epistémicas. Gran parte de este trabajo sugiere una erosión de la autoridad de los estados-nación como las principales unidades de análisis a nivel internacional.

Normas e ideas transnacionales: El surgimiento de normas e ideas transnacionales es importante para generar una cooperación duradera. Se genera mediante la cooperación y se transmite a la política interna. Esta literatura minimiza la riqueza y poder económico para determinar la naturaleza y el alcance de la cooperación internacional. Los investigadores apuntan a una comprensión más constructivista de cómo los estados y las ANE interactúan y aprenden unos de otros, y cómo se forman las preferencias e identidades de los actores. Eficacia del régimen: Los efectos de la cooperación recaen sobre la política internacional. Las preguntas en este campo de estudio se refieren a qué tan bien los estados cumplen con los acuerdos, qué medidas toman para implementarlos y hasta qué punto los acuerdos o regímenes resuelven realmente los problemas para los que fueron diseñados (O’Neill., et al, 2003)

Las diferentes formas de cooperación enmarcan a la cooperación internacional en el ámbito del desarrollo para los países en vías de desarrollo y fortalecimiento de aquellos que ya han alcanzado niveles de desarrollo e industrialización de manera previa. Es decir, se observa que, en el desglose de las relaciones internacionales, el sistema internacional hasta llegar a la cooperación internacional enfocada en el desarrollo, se puede mencionar que éstos inciden en el flujo del comercio internacional del Ecuador con otros países de la Región. Es decir, el flujo del comercio internacional del Ecuador con otros países de la Región podría analizarse desde su calidad de miembro de la Comunidad Andina de Naciones y desde su calidad de Estado Asociado del Mercosur.Sin embargo, es importante mencionar que existen pocos estudios sistemáticos al respecto del papel que la Cooperación Internacional para el Desarrollo desempeña en la configuración del sistema internacional o en la economía mundial (González, 2011).

No obstante, esta investigación pretende conocer como desde la CID, el Ecuador ha vivido el flujo de su comercio internacional con sus pares de la región (CAN y MERCOSUR), considerando además que estos dos bloques de América Latina cuentan con un acuerdo de cooperación, cuyas etapas son las siguientes: La integración CAN-MERCOSUR es el resultado de un proceso que puede dividirse en tres etapas: la primera de negociaciones que comienza con la creación del MERCOSUR en 1995 y se consolida con el Acuerdo Marco de Integración de 1998 con la Comunidad Andina de Naciones CAN; la segunda de firma del Acuerdo de Complementación Comercial ACE 59 del 2004 CAN-MERCOSUR; y una tercera de desarrollo del programa de desgravación, que enmarca la evolución y las tensiones del nuevo escenario político regional anti Norteamericano y la firma del Tratado de Liberación Comercial Colombia-Estados Unidos. La CID considerada por su contenido, puede ser de carácter general o sectorial, orientada a la toma de decisiones conjuntas, al establecimiento de normas internacionales o de carácter operativo en laimplementación de medidas o programas concretos de desarrollo. También puede ser, según su grado de institucionalización, de carácter informal u orgánico, en el seno o como consecuencia de la actividad de Organizaciones Internacionales.

El concepto de cooperación internacional se ha utilizado en la literatura sobre relaciones internacionales. Una definición estándar es que la cooperación ocurre cuando los actores ajustan su comportamiento a las preferencias reales o anticipadas de los demás. Esta cooperación describe interacciones para lograr objetivos comunes cuando las preferencias de los actores no son idénticas pero tratan de logar armonía a pesar del conflicto a nivel multilateral, regional y global (Paulo, 2014). La cooperación puede darse a través de tres dimensiones: contenido sustantivo, participación amplia y legalización. En cuanto al contenido sustantivo, éste impulsa la idea que los estados pueden comprometerse en ausencia de un acuerdo formal y jurídicamente vinculante. Dependiendo de la naturaleza del problema, los acuerdos requieren diferentes niveles de participación para ser efectivos.

En referencia a la legalización, ésta combina elementos normativos, es decir, muchos acuerdos internacionales están altamente legalizados bajo la forma de tratados, por ejemplo, por lo que se consideran vinculantes en cuestión de derecho internacional para los Estados que los aceptan (Abbott \& Snidal, 2004). Así mismo en el comercio internacional sus fundamentos están relacionados con el abastecimiento al menor costo posible y sus principios han servido para orientar los objetivos de la política comercial de los países.

La cooperación ayuda a los estados a obtener beneficios mutuos, pero la desconfianza y los desacuerdos sobre el diseño institucional inhiben la cooperación. Se enfatiza en los acuerdos bilaterales que constituyen una red de vínculos de cooperación en evolución. Esta red define el entorno estratégico en el que los estados 
negocian sobre nuevos acuerdos, influyendo endógenamente en los esfuerzos bilaterales posteriores al revelar información estratégicamente valiosa sobre la confiabilidad y las preferencias de los estados sobre el diseño institucional, al tiempo que genera externalidades que incentivan las asociaciones bilaterales. Por lo que es más probable que los estados creen acuerdos bilaterales si (1) comparten acuerdos con terceros comunes, (2) acceden a más acuerdos en general y / o (3) comparten características exógenas importantes con socios bilaterales actuales. Estas dinámicas de red impulsan la cooperación bilateral en todo, desde los productos básicos hasta el intercambio cultural y la pesca. (Kinne, 2013). La cooperación internacional plantea desafíos para superar la fragmentación, la falta de compromiso y la sectorización a nivel nacional e internacional y global, por lo que se requieren del impulso de foros que tengan el potencial de influir en el marco de esta cooperación para lograr la implementación de una agenda global, como por ejemplo el G20. (Messner, et al., 2013)

\section{METODOLOGÍA}

El diseño de investigación es no experimental ya que no se controlan factores externos y al modelar series de tiempo no se incorporan conceptos de aleatoriedad en sus datos. Así mismo la lógica de la investigación resultó ser deductiva dado que existe un componente teórico que soporta la relación entre el dinamismo comercial internacional y los acuerdos internacionales entre países. En consecuencia, la investigación desarrollada enmarca su desarrollo en un diseño no experimental y una lógica deductiva.

El enfoque de la investigación se considera cuantitativo dado que se usaron técnicas econométricas de serie de tiempo para poder observar cambios significativos. El alcance de la investigación resultó ser correlacional, lo cual permitió observar cambios en el dinamismo comercial producto acuerdos comerciales entre países. Para el análisis de datos se utilizó datos desde el año 1997 hasta el periodo 2019 registrando un total de 23 observaciones. Los datos recogidos fueron las exportaciones e importaciones de países miembros de la CAN, entre estos Ecuador, Perú, Bolivia y Colombia. Los datos recogidos provienen de fuentes oficial, entre estas Banco Central del Ecuador y Banco mundial. Los valores registrados fueron dólares de exportaciones e importaciones en FOB.

Para modelar la significancia del crecimiento del flujo comercial entre Ecuador y los países miembros de la CAN se consideraron dos perspectivas. La primera perspectiva analizó el cambio significativo que pudieron tener las exportaciones de Ecuador hacia países miembros de la CAN. La segunda perspectiva consideró el cambio significativo que puedan tener las importaciones de Ecuador que provienen de países miembro de la CAN.Para modelar las series se consideró un modelo ARMAX Auto-Regressive Moving Average with exogenous inputs. El modelo propuesto parte de un modelo Arma. Los modelos finalmente expuestos a través del programa Gretel fueron desarrollados bajo la siguiente lógica:

$\mathrm{y}_{\mathrm{t}}=$ const $+\emptyset_{1}$ phi $_{1}+\emptyset_{2}$ theta $_{2}+\emptyset_{3}$ time $+\mathrm{w}_{\mathrm{t}}$

Siendo $\emptyset 1, \varnothing 2, \varnothing 3$ los coeficientes de las variables explicativas del modelo ARMAX de serie de tiempo. Las variables Phi, Theta_1 y Time hacen referencia a las variables explicativas de ajuste del modelo de serie de tiempo. Las hipótesis planteadas al modelo fueron las siguientes:1) H1: El dinamismo comercial entre Ecuador y los países miembros de la CAN ha sido significativo; 2) H1a: El dinamismo de las importaciones de Ecuador proveniente de países miembros de la CAN ha sido significativo; y 3) $\mathrm{H} 1 \mathrm{~b}$ : El dinamismo de las exportaciones de Ecuador hacia países miembros de la CAN ha sido significativo.

\section{RESULTADOS}

A continuación, se presenta cada uno de los análisis realizados basados en modelos econométricos para explicar relaciones importantes entre las variables de estudio.

\section{Modelo de importaciones}

Se observa en la tabla 1 la modelación de serie de tiempo para un modelo Armax de importaciones de Bolivia donde se observa que las variables predictores para el modelo de serie de tiempo son significativas excepto el ajuste theta_1. Adicional, en la tabla 2, se realizó un test de normalidad de residuos donde se pudo observar que los errores se ajustaban a una distribución normal. En la tabla 3, el modelo de serie de tiempo de importaciones provenientes de Colombia se puede observar que las variables explicativas para el modelo ARMAX resultaron significativas al 1\% de significancia, los coeficientes de la misma muestran una relación directa. Así mismo se demostró que los residuos del modelo se ajustan a una distribución normal. Las importaciones provenientes de Perú fueron modeladas en serie de tiempo donde se observar que sus variables explicativas resultaron significativas para el modelo, así mismo se realizó una verificación de supuestos pudiendo concluir que los errores se ajustan a una distribución normal. 
Tabla 1: Modelo 1 ARMAX, usando las observaciones 1997-2019 (T=23)

\begin{tabular}{|c|c|c|c|c|c|}
\hline \multicolumn{6}{|c|}{ Variable dependiente: Bolivia } \\
\hline \multicolumn{6}{|c|}{ Desviaciones típicas basadas en el Hessiano } \\
\hline & Coeficiente & Desv. Típica & z & valor $p$ & \\
\hline const & -40.0271 & 456.722 & -0.8764 & 0.0018 & $* * *$ \\
\hline phi_1 & 0.865224 & 0.104950 & 8.244 & $<0.0001$ & $* * *$ \\
\hline theta_1 & 0.0328440 & 0.186512 & 0.1761 & 0.8602 & \\
\hline time & 989.272 & 283.612 & 3.488 & 0.0005 & $* * *$ \\
\hline \multicolumn{2}{|c|}{ Media de la vble. dep. } & 6.112 .511 & \multicolumn{2}{|c|}{ D.T. de la vble. dep. } & 8.338 .577 \\
\hline \multicolumn{2}{|c|}{ Media de innovaciones } & -1.888457 & \multicolumn{2}{|c|}{ D.T. innovaciones } & 2.228 .339 \\
\hline \multicolumn{2}{|c|}{ Log-verosimilitud } & -104.7429 & \multicolumn{2}{|c|}{ Criterio de Akaike } & 2.194 .858 \\
\hline \multicolumn{2}{|c|}{ Criterio de Schwarz } & 2.251 .632 & \multicolumn{2}{|c|}{ Crit. de Hannan-Quinn } & 2.209 .136 \\
\hline
\end{tabular}

Tabla2: Modelo 2 ARMAX, usando las observaciones 1997-2019 (T = 23)

\begin{tabular}{|l|c|c|c|c|c|}
\hline \multicolumn{5}{|c|}{ Variable dependiente: I_Colombia } \\
\hline & Coeficiente & Desv. TÃ-pica & $z$ & valor p & \\
\hline const & 6.27494 & 0.151991 & 41.28 & $<0.0001$ & ${ }^{* * *}$ \\
\hline theta_1 & 0.686204 & 0.126963 & 5.405 & $<0.0001$ & ${ }^{* * *}$ \\
\hline time & 0.0707807 & 0.0109795 & 6.447 & $<0.0001$ & ${ }^{* * *}$ \\
\hline Media de la vble. dep. & 7.140558 & D.T. de la vble. dep. & 0.564542 \\
\hline Media de innovaciones & 0.006015 & D.T. innovaciones & 0.217858 \\
\hline Log-verosimilitud & 2.096107 & Criterio de Akaike & 3.807786 \\
\hline Criterio de Schwarz & 8.349763 & Crit. de Hannan-Quinn & 4.950080 \\
\hline
\end{tabular}

Tabla 3: Modelo 3 ARMAX, usando las observaciones 1997-2019 (T = 23)

\begin{tabular}{|c|c|c|c|c|c|}
\hline \multicolumn{6}{|c|}{ Variable dependiente: I_Peru } \\
\hline \multicolumn{6}{|c|}{ Desviaciones típicas basadas en el Hessiano } \\
\hline & Coeficiente & Desv. Típica & $z$ & valor $p$ & \\
\hline const & 4.23579 & 0.440980 & 9.605 & $<0.0001$ & *** \\
\hline phi_1 & 0.837902 & 0.122612 & 6.834 & $<0.0001$ & $\star \star \star *$ \\
\hline theta_1 & 0.0373972 & 0.204908 & 0.1825 & 0.0052 & *** \\
\hline time & 0.126819 & 0.0296109 & 4.283 & $<0.0001$ & $\star \star \star *$ \\
\hline \multicolumn{2}{|c|}{ Media de la vble. dep. } & 5.903438 & \multicolumn{2}{|c|}{ D.T. de la vble. dep. } & 1.073829 \\
\hline \multicolumn{2}{|c|}{ Media de innovaciones } & 0.013807 & \multicolumn{2}{|c|}{ D.T. innovaciones } & 0.264028 \\
\hline \multicolumn{2}{|c|}{ Log-verosimilitud } & â^’2.580786 & \multicolumn{2}{|c|}{ Criterio de Akaike } & 15.16157 \\
\hline \multicolumn{2}{|c|}{ Criterio de Schwarz } & 20.83904 & \multicolumn{2}{|c|}{ Crit. de Hannan-Quinn } & 16.58944 \\
\hline
\end{tabular}

\section{Modelo de exportaciones}

Se observa en la tabla 4, la modelación de serie de tiempo para un modelo Armax de exportaciones a Bolivia donde se observa que las variables predictores para el modelo de serie de tiempo son significativas excepto el ajuste theta_1. Adicional a esto, en la tabla 5, se realizó un test de normalidad de residuos donde se pudo observar que los errores se ajustaban a una distribución normal.

Tabla 4: Modelo 4 ARMAX, usando las observaciones 1997-2019 (T = 23)

\begin{tabular}{|l|c|c|c|c|c|}
\hline \multicolumn{5}{|c|}{ Vesviaciones típicas basadas en el Hessiano } \\
\hline & Coeficiente & Desv. Típica & $z$ & valor $p$ & \\
\hline const & -0.839665 & 3.32190 & -0.2528 & 0.0084 & ${ }^{* *}$ \\
\hline phi_1 & 0.719554 & 0.298026 & 2.414 & 0.0158 & ${ }^{* *}$ \\
\hline theta_1 & -0.372977 & 0.356047 & -1.048 & 0.2948 & \\
\hline time & 1.57579 & 0.219936 & 7.165 & $<0.0001$ & ${ }^{* *}$ \\
\hline Media de la vble. dep. & 17.48573 & D.T. de la vble. dep. & 11.89041 \\
\hline Media de innovaciones & -0.219091 & D.T. innovaciones & 3.860889 \\
\hline Log-verosimilitud & -63.83329 & \multicolumn{5}{l|}{ Criterio de Akaike } & 137.6666 \\
\hline
\end{tabular}


Tabla 5: Modelo 5 ARMAX, usando las observaciones 1997-2019 ( $T=23)$

\begin{tabular}{|c|c|c|c|c|c|}
\hline \multicolumn{6}{|c|}{ Variable dependiente: Colombia } \\
\hline \multicolumn{6}{|c|}{ Desviaciones típicas basadas en el Hessiano } \\
\hline & Coeficiente & Desv. Típica & $z$ & valor $p$ & \\
\hline const & 283.740 & 109.952 & 2.581 & 0.0099 & *** \\
\hline phi_1 & 0.621489 & 0.201772 & 3.080 & 0.0021 & $* * *$ \\
\hline theta_1 & 0.264966 & 0.235005 & 1.127 & 0.2595 & \\
\hline time & 29.5862 & 7.84352 & 3.772 & 0.0002 & *** \\
\hline \multicolumn{2}{|c|}{ Media de la vble. dep. } & 642.8558 & \multicolumn{2}{|c|}{ D.T. de la vble. dep. } & 268.9770 \\
\hline \multicolumn{2}{|c|}{ Media de innovaciones } & â^1.879155 & \multicolumn{2}{|c|}{ D.T. innovaciones } & 89.64254 \\
\hline \multicolumn{2}{|c|}{ Log-verosimilitud } & $\hat{a ̂ A}^{\wedge} 136.4726$ & \multicolumn{2}{|c|}{ Criterio de Akaike } & 282.9452 \\
\hline \multicolumn{2}{|c|}{ Criterio de Schwarz } & 288.6227 & \multicolumn{2}{|c|}{ Crit. de Hannan-Quinn } & 284.3731 \\
\hline
\end{tabular}

En la tabla 6, el modelo de serie de tiempo de exportaciones a Colombia, se puede observar que las variables explicativas para el modelo ARMAX resultaron significativas al 1\% de significancia excepto theta_1, los coeficientes de la misma muestran una relación directa. Así mismo se demostró que los residuos del modelo se ajustan a una distribución normal. Las exportaciones a Perú fueron modeladas en serie de tiempo donde se observar que sus variables explicativas resultaron significativas para el modelo excepto Theta_1, así mismo se realizó una verificación de supuestos pudiendo concluir que los errores se ajustan a una distribución normal.

Tabla 6: Modelo 6 ARMAX, usando las observaciones 1997-2019 ( $T=23)$

\begin{tabular}{|c|c|c|c|c|c|}
\hline \multicolumn{6}{|c|}{ Variable dependiente: I_Peru } \\
\hline \multicolumn{6}{|c|}{ Desviaciones tã-picas basadas en el Hessiano } \\
\hline & Coeficiente & Desv. TÃ-pica & \begin{tabular}{l|l} 
& $z$ \\
\end{tabular} & valor $p$ & \\
\hline const & 5.65420 & 0.412811 & 13.70 & $<0.0001$ & *** \\
\hline phi_1 & 0.727302 & 0.202604 & 3.590 & 0.0003 & *** \\
\hline theta $\_1$ & 0.146633 & 0.340184 & 0.4310 & 0.6664 & \\
\hline time & 0.0766106 & 0.0289856 & 2.643 & 0.0082 & 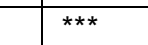 \\
\hline \multicolumn{2}{|c|}{ Media de la vble. dep. } & 6.688740 & \multicolumn{2}{|c|}{ D.T. de la vble. dep. } & 0.770568 \\
\hline \multicolumn{2}{|c|}{ Media de innovaciones } & 0.012882 & \multicolumn{2}{|c|}{ D.T. innovaciones } & 0.294878 \\
\hline \multicolumn{2}{|c|}{ Log-verosimilitud } & â^5.036755 & \multicolumn{2}{|c|}{ Criterio de Akaike } & 20.07351 \\
\hline \multicolumn{2}{|c|}{ Criterio de Schwarz } & 25.75098 & \multicolumn{2}{|c|}{ Crit. de Hannan-Quinn } & 21.50138 \\
\hline
\end{tabular}

\section{DISCUSIÓN}

A nivel internacional los estudios de comercio internacional tienden a centrarse su objeto de estudio en los acuerdos y tratados comerciales dada la importancia de estos para generar competitividad y evidenciar ventajas comparativas entre países. Uno de los casos más importantes es el dinamismo del comercio internacional representado y evidenciado por países de la CAN. Los resultados obtenidos muestran la importancia de la presencia de acuerdos comerciales para dinamizar el comercio internacional. El modelo ARMAX utilizado ajustó el comportamiento del flujo comercial registrado entre ecuador y países miembros de la CAN. Se observa un dinamismo comercial creciente y significativo entre Ecuador y países miembros de la CAN como Perú, Colombia y Bolivia. Los pronósticos obtenidos permitieron entender la significancia en los cambios de las variables observadas a lo largo del tiempo, así mismo los pronósticos permitieron anticiparse a los niveles de flujo de comercio internacional en el futuro.

Otros Autores sugieren que los acuerdos comerciales son realmente importantes para poder influenciar a la apertura comercial de los países y esto a su vez podría impactar en el bienestar económico de una nación. La metodología aplicada en este estudio presenta robustez y permite crear pronósticos adecuados sin embargo otros autores podrían aplicar otras técnicas econométricas que permitan medir la importancia de los acuerdos comerciales entre países de la comunidad. Así mismo se recomienda realizar futuras investigaciones relacionadas a la importancia y efectos macroeconómicos de los tratados de libre comercio midiendo impactos en el corto, mediano y largo plazo.El comercio internacional ha generado interés por investigadores a nivel mundial y en muchas ocasiones sus estudios son abordados bajo una perspectiva macroeconómico o microeconómica. En este estudio las perspectivas de los resultados fueron abordados bajo una visión macroeconómica de las variables de estudio. Uno de los principales hallazgos y conclusiones radica en que las variables exportaciones e importaciones presentan un dinamismo entre países de la CAN y esto permite anticipar la importancia de los acuerdos comerciales. 
Las exportaciones y las importaciones de Ecuador con países miembros del a CAN han tenido un dinamismo significativo lo cual se puede observar en el comportamiento de los modelos de serie de tiempo aplicados. Así mismo se puede evidenciar la evolución importante que ha tenido el comercio internacional de Ecuador con miembros de países de la CAN dando relevancia a la presencia de los acuerdos comerciales para generar impactos significativos en una economía. El flujo de importaciones ha aumentado fuertemente por parte del Ecuador lo que se pronostica que la tendencia se mantenga a la alta pues los valores de la constante e intercepto de los modelos se mantienen significantes, hay normalidad residual y los módulos son valores por encima de 1 por lo que se confirma que el mejor modelo para estudiar este comportamiento fue el modelo ARIMA, dando como resultado como primer lugar las importaciones desde Colombia, seguido por Perú y terminando con Bolivia.

En el Ecuador el flujo de exportaciones no deja de crecer pero sin superar las importaciones, lo que se pronostica que la tendencia se mantenga a la alta pues los valores de la constante e intercepto de los modelos se mantienen significantes, hay normalidad residual y los módulos son valores por encima de 1 por lo que se confirma que el mejor modelo para estudiar este comportamiento fue el modelo ARIMA, dando como resultado como primer lugar las exportaciones hacia Perú, seguido por Colombia y terminando con Bolivia. Finalmente se puede evidenciar la importancia de los acuerdos comerciales para poder impactar en el bienestar de una sociedad teniendo en cuenta la balanza comercial juega un papel fundamental en la estimación del producto interno bruto y en los saldos de balanza de pago de cuenta corriente, siendo este último un factor importante de análisis en una economía dolarizada.

\section{CONCLUSIONES}

De acuerdo al trabajo presentado y a los resultados obtenidos, se pueden plantear las siguientes conclusiones principales:

1. Las relaciones internacionales juegan un rol fundamental en la economía de un país de tal forma que promueve la competitividad y el intercambio eficiente de bienes y servicios. Esta evolución en el intercambio de servicios se pudo evidenciar en los resultados obtenidos de tal forma que las exportaciones e importaciones registraron dinamismos. En consecuencia, tanto teórico como empíricamente se pudo observar impactos en el comercio internacional.

2. Se pudo observar el flujo de apertura comercial entre países miembros de la CAN han tenido un dinamismo significativo lo cual da relevancia a este tipo de acuerdos. Asi mismo los niveles de importación de Ecuador han mostrado volatilidad y una tendencia creciendo lo cual puede provocar inestabilidad en los saldos de la cuenta comercial. Por consecuente es necesario fortalecer el dinamismo de exportaciones para tratar de generar una posición de ventaja comercial que permita ingreso de divisas y sostenga la dolarización en Ecuador.

3. En el contexto actual se puede evidenciar impactos de las relaciones internacionales, lo cual se ve reflejado en la transferencia de bienes y servicios de calidad que, asi como un aumento en los niveles de competitividad de los productos locales.

\section{REFERENCIAS}

Abbott, K., y Snidal, D., Pathways to international cooperation, https://dx.doi.org/10.1017/CBO9780511494147.003, The Impact of International Law on International Cooperation: Theoretical Perspectives, 54(3), 50-84 (2004)

Allard, R., Tendencias y nuevas temáticas en el mundo global: desafíos para Chile y América Latina, Estudios Internacionales, 165(1), 75-108 (2010)

Amunátegui, C., y Carvajal, P., Hacia una protohistoria del comercio en el creciente fértil y su interconexión con el derecho, https://dx.doi.org/10.4067/S0716-54552016000100001, Revista de Estudios Históricos-Jurídicos, 38(1), 37-45 (2016)

Caruncho, M.I., Jaime, P., y Castillo, M., Acabar con la pobreza: un reto para la cooperación internacional, 3를 Ed., 1-290, Fundación IPADE, Madrid, España (2003)

Bhansali, K., Neorealism and its Critics, https://doi.org/10.2307/2131329, The Journal of Politics, 49(2), $631-633$ (1987)

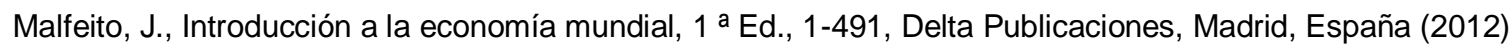

Coronado, J. L., ¿Qué es la Globalización? Falacias del globalismo, respuestas a la globalización, Frontera Norte, 13(25), 155-163 (2001)

Browne, S., The raise and fall of development aid, World Institute for Development Economics Research, 1(143), 1-37 (1997) 
Cornago, N., Desarrollo, Subdesarrollo y Post-desarrollo, Un análisis crítico del debate contemporáneo, 1ํa Ed., 39-88, Editorial Bilbao, Madrid, España (1998)

Friedrichs, J., An intercultural theory of international relations: how self-worth underlies politics among nations, https://doi.org/10.1017/S1752971915000202, International Theory, 8(1), 63-96 (2016)

González, R., Diferentes teorías del comercio internacional, Revista de Economía, 1(858), 103-118 (2011)

Haas, E. B., Does Constructivism Subsume Neo-Functionalism?, 1ª Ed., 437-446, Macmillan, Basingstoke, Hampshire (2006)

Held, D., Democracia y Orden Global del Estado Moderno al Gobierno Cosmopolita,1ª Ed., 23-371, Paidós, Barcelona, España (1997)

Hinojosa, L. M., Globalización y Soberanía de los Estados, Revista Electrónica de Estudios Internacionales, 1(10), 1-14 (2005)

Hoffmann, S., El estado de las cosas, Vanguardia Dossier, 1(3), 20-30 (2002)

Holsti, K.J., International Politics: A Framework for Analysis, $1^{\text {a }}$ Ed., 1-432, Prentice Hall, United States of America (1983)

Iglesia, M., El impacto económico y social de la cooperación para el desarrollo, $1^{\mathfrak{a}}$ Ed., Universidad Complutense de Madrid, Madrid, España (2005)

Keohane, R., y Nye, J., Globalization: What's New? What's Not? (And So What?), https://doi.org/10.2307/1149673, Foreign Policy, 1(118), 104-119 (2000)

Kinne, B., Network Dynamics and the Evolution of International Cooperation, https://doi.org/10.1017/S0003055413000440, American Political Science Review, 107(4), 766-785 (2013)

Lawson, S., Theories of International Relations, $1^{\text {a }}$ Ed., 1-288, Wiley, Sidney, Australia (2015)

Messner, D., Guarin, A., y Haun, D., The behavioural dimensions of international cooperation, https://dx.doi.org/10.2139/ssrn.2361423, Centre for Global Cooperation, 1(1), 1-42 (2013)

Meyer, T., Globalization, Regionalization and Stateness, $1^{\text {a }}$ Ed, 119-136, Springer, Dordrecht, Netherland (2012)

Molano, G., El interregionalismo y sus límites, Estudios Internacionales, 1(158), 9-27 (2007)

O’Neill, K., Balsiger, J., y otro autor, Actors, Norms, and Impact: Recent International Cooperation Theory and the Influence of the Agent-Structure Debate, https://doi.org/10.1146/annurev.polisci.7.090803.161821, Annual Review of Political Science, 14(7), 75-149 (2003)

Paulo, S., International cooperation and development: A conceptual overview, https://dx.doi.org/10.2139/ssrn.2430206, German Development Institute, 14(13), 1-31 (2014)

Palomares, G., Relaciones internacionales en el siglo XXI, 2ª Ed., 1-227, Editorial Tecnos, Madrid, España (2006)

Rogerson, A., Hewitt, A., y Waldenberg, D., The International Aid System 2005 - 2010: Forces For and Against Change, 1a Ed., 1-32, Oversea Development Institute, Londres, Inglaterra (2004)

Rodríguez, I., y Sotillo, J.A., Relaciones Internacionales, Política Exterior y Cooperación para el Desarrollo: reflexiones para un debate en el caso español, Revista Española de Derecho Internacional, 55(1), 149-186 (2003)

Silva, C., Globalización: Dimensiones y políticas públicas, Hologramática, 1(10), 3-25 (2009)

Van De Mieroop, M., In Search of Prestige: foreign Contacts and the Rise of an Elite in Early Dynastic Babylonia, $1^{\text {a }}$ Ed., 125-137, Eisenbrauns, Winona Lake, Indiana (2002) 
